\title{
Learning from evolving data streams
}

\author{
Jacob Montiel
}

University of Waikato

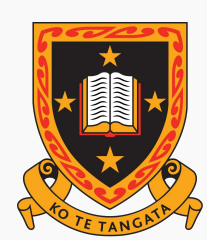

SciPy2020 


\section{Standard machine learning}

- Based on data batches (batch learning)

- State-of-the-art performance on multiple applications

- Batch learning pipeline:

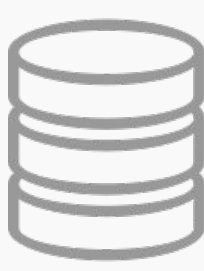

data

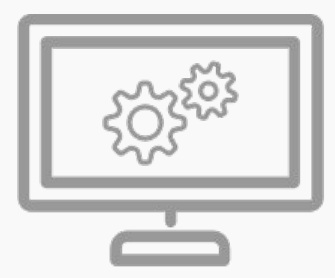

training

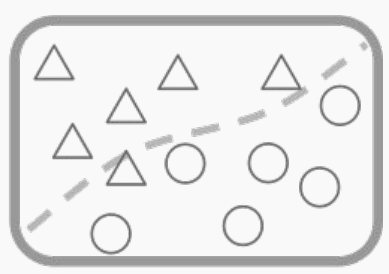

model 


\section{Standard machine learning}

- Based on data batches (batch learning)

- What if data...

a. is continuously generated (not available at once)

b. changes over time
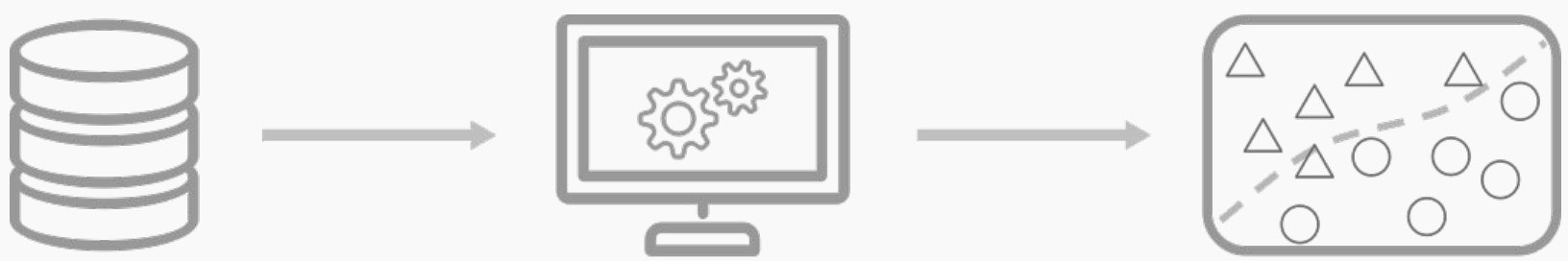

data

training

model 


\section{Example: Supply chain}

\section{Number of Top 10 Amazon search terms* related to the Coronavirus}

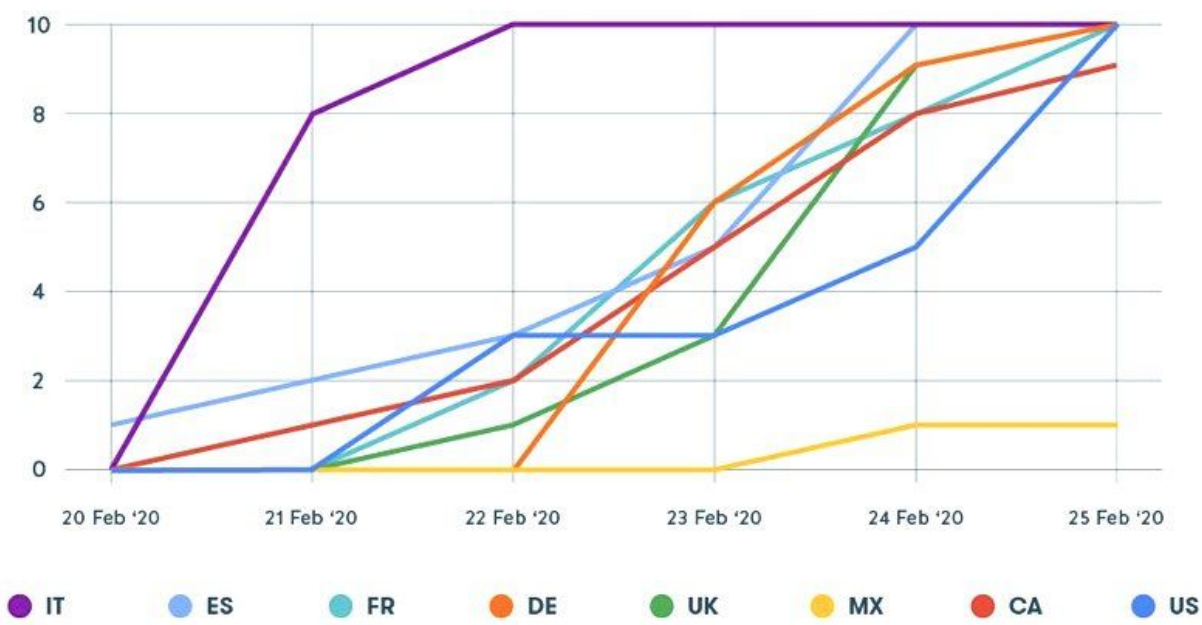

"It took less than a week at the end of February for the top 10 Amazon search terms in multiple countries to fill up with products related to covid-19."

"Our weird behavior during the pandemic is messing with Al models". Will Douglas Heaven. MIT Technology Review. May 11, 2020 


\section{Stream learning}

- Unbounded training sets

- Data is assumed infinite

- Maintain models in an online fashion
- Incorporate data on the fly

- $\quad$ Resource-wise efficient

- Detect changes and adapt

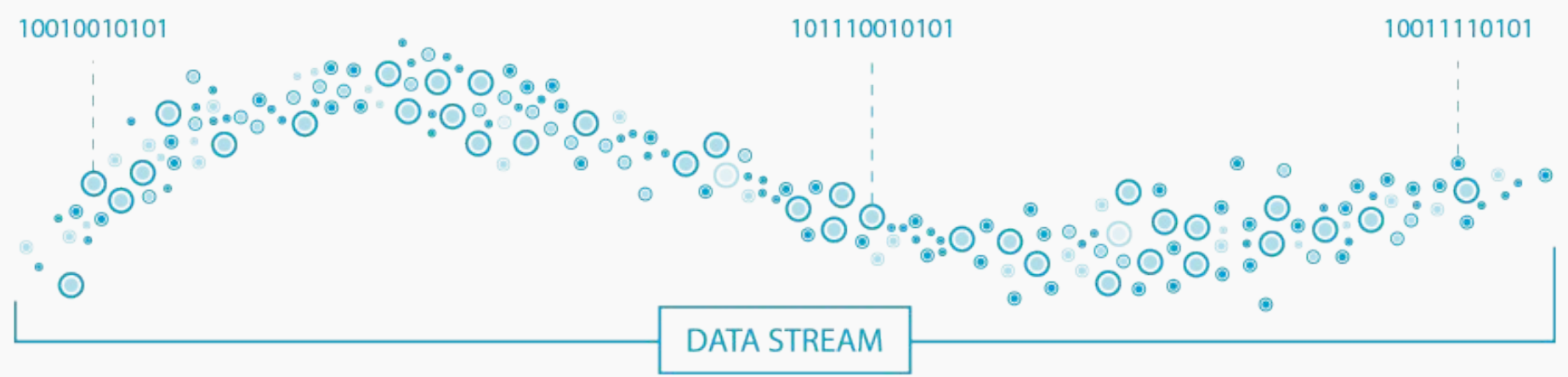




\section{Requirements}

Q Process one sample at a time, and inspect it only once

[]. Use a limited amount of memory

(D) Work in a limited amount of time Always ready to predict 


\section{Learning from data streams}

Supervised learning

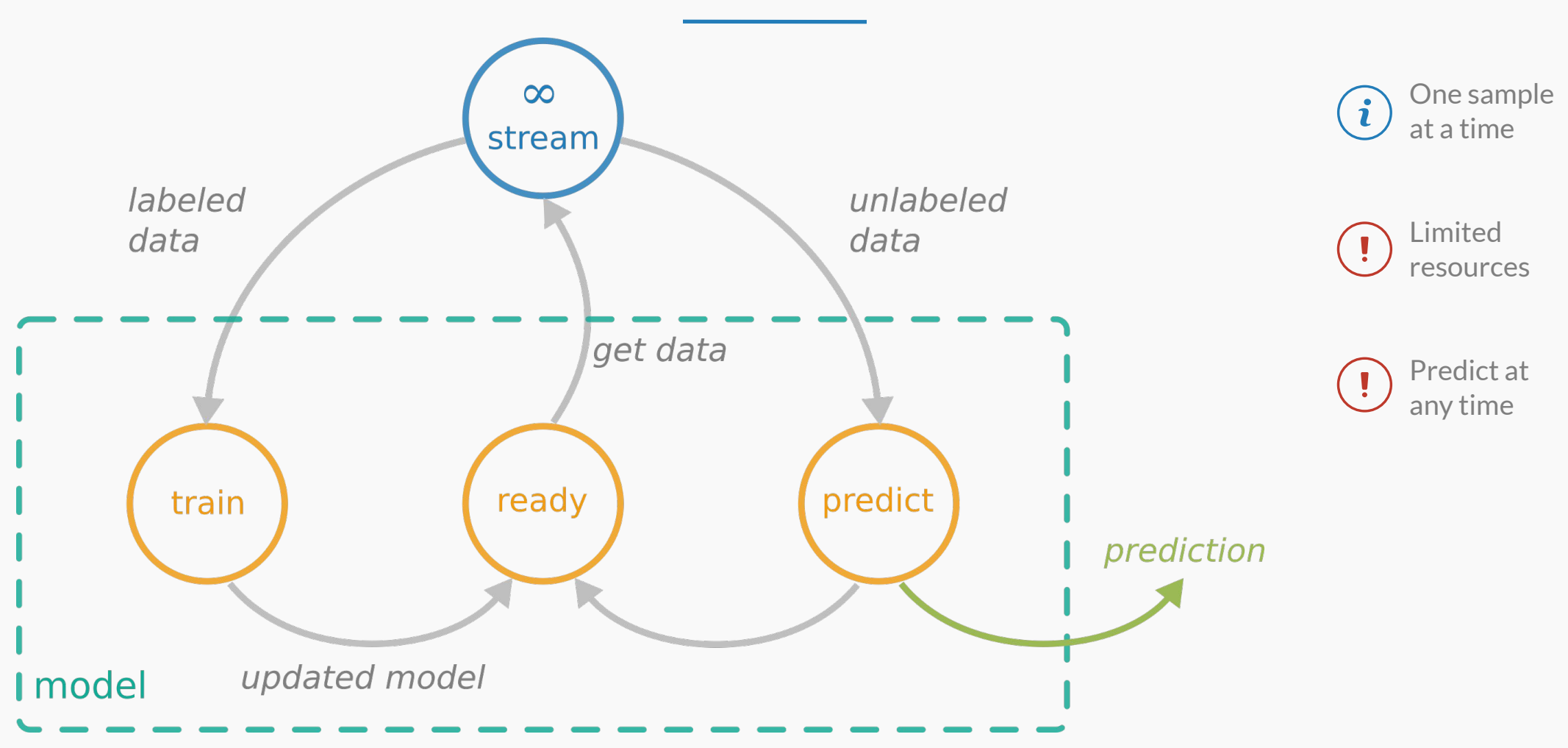




\section{Decision Tree Classifier}

Example: Buying an used car

- Popular batch method

- Good performance + interpretability

- Greedy recursive induction

- Sort all instances through tree

- $x_{\mathrm{i}}=$ most discriminative attribute

- New split node for $x_{i}$

new branch for each value

leaf node assigns class

- Stop if no error or limit on \#instances

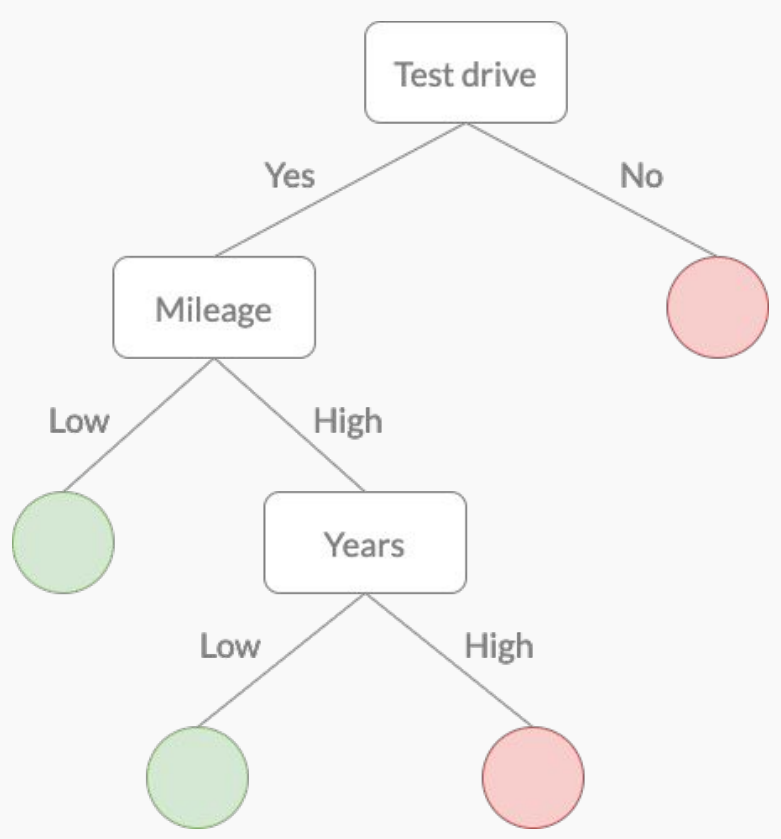




\section{Very Fast Decision Tree}

a.k.a. Hoeffding Tree

- Incrementally expand (split) nodes

- A small sample can often be enough to choose a near optimal decision

- Collect (sufficient) statistics

$$
t_{1}=t_{0}+\delta_{1}
$$

- Estimate the merit of each attribute

- Choose the sample size that allows to differentiate between the alternatives

Hoeffding bound 


\section{Very Fast Decision Tree}

a.k.a. Hoeffding Tree

- The number of examples to expand a node depends

$$
\begin{aligned}
& t_{0} \\
& t_{1}=t_{0}+\delta_{1}
\end{aligned}
$$

only on the Hoeffding bound

- error decreases as more data is observed

- Popular stream method

- Low variance

- Low overfitting

- Asymptotically close to the batch model

$$
t_{2}=t_{1}+\delta_{2}
$$




\section{Concept drift}

In dynamic and non-stationary environments, the data distribution can change over time

- Change detection: Given an input sequence $\left\langle x_{1}, x_{2}, \ldots, x_{t}, \ldots\right\rangle$ raise an alarm signal at instant $t$ if there is a distribution change

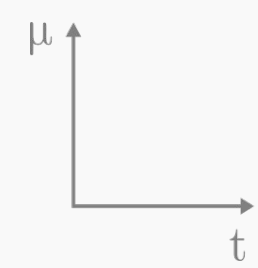

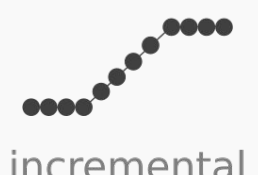

abrupt

- Application: Detect changes in model performance

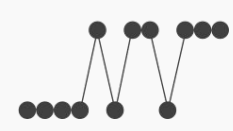

gradual

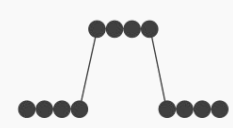

recurring

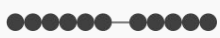

outlier 


\section{ADWIN change detector}

ADaptive WINdowing

- Adaptive window with two subwindows

- Rise an alarm if subwindows exhibit "distinct enough" averages

- Subwindows are recomputed online according to the rate of change

- Theoretical guarantees

- Logarithmic memory and update time adaptive window with two subwindows
1. iteration $2121420120 \ldots 121300$
2. iteration $2221420120 \ldots 121300$
: ${ }_{\text {iteration }}=$ cut check position :
n. iteration $221420120 \ldots 12 n 1300$ 


\section{Learning from evolving data}
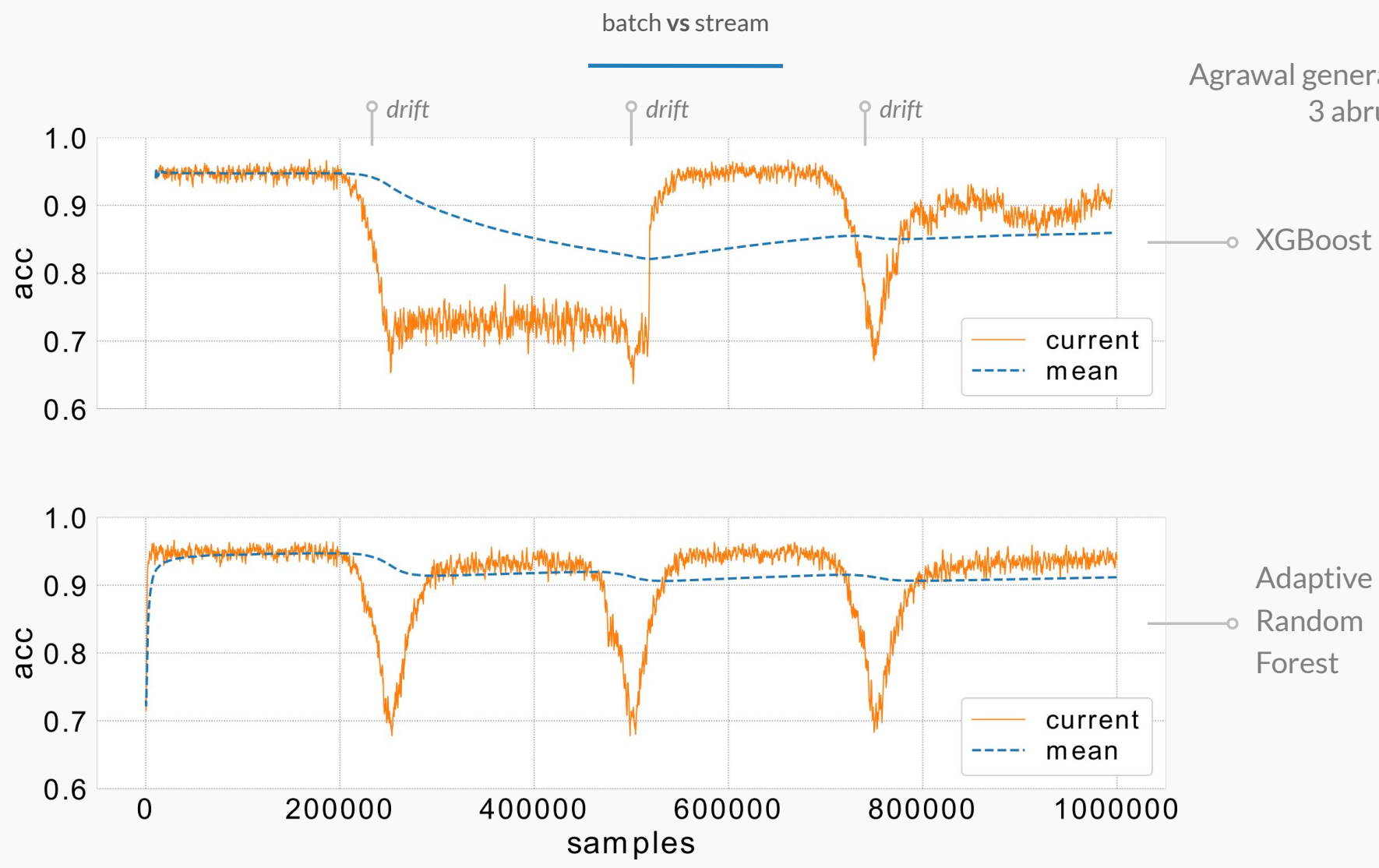


\section{Evaluation}

Holdout an independent test set

- Apply the current model to the test set, at regular time intervals

- Unbiased performance estimation

- Popular in batch and stream learning

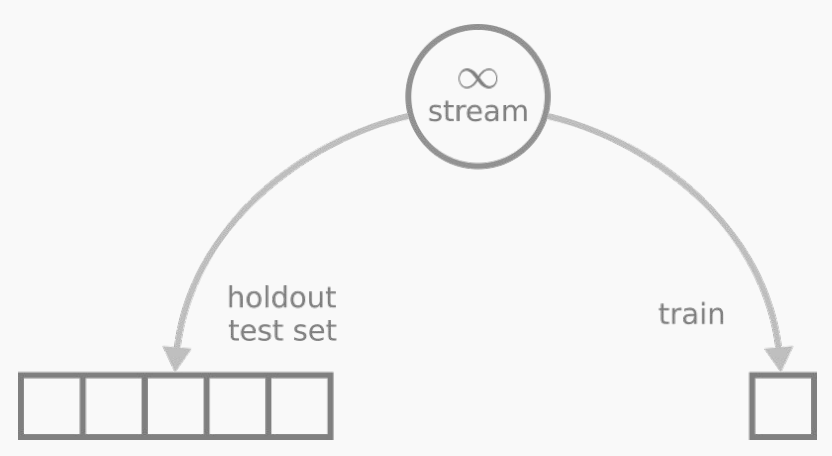

Prequential

- Test then train each new instance

- Order matters!

- All data is used for training

- Performance is estimated on the sequence

- Popular in the stream setting

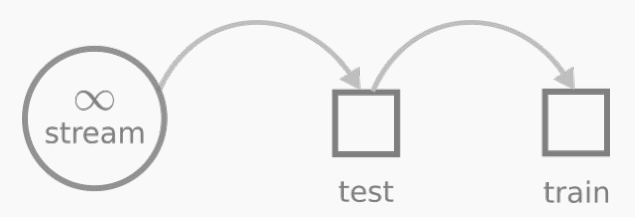




\section{scikit-multiflow}

A machine learning package for data streams in Python

- Easy to design and run experiments

- Contains

- Easy to extend existing methods

- For users with any experience level

- Low learning curve

- Works in Jupyter Notebooks
- data generators
o stream learning methods
$\circ \quad$ change detectors
- evaluators
○ and more
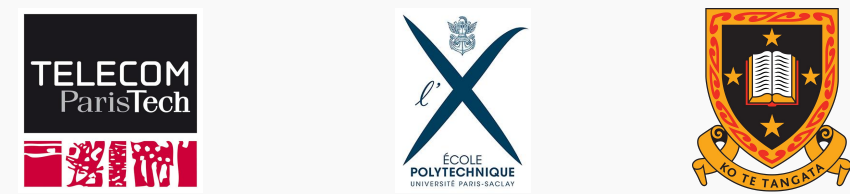


\section{Get scikit-multiflow}

Multiple sources available

- scikit-multiflow works on Linux, macOS and Windows

- Recommended:

- conda-forge

$\$$ conda install -c conda-forge scikit-multiflow

- PYPI

\$ pip install scikit-multiflow

- GitHub (latest development version)

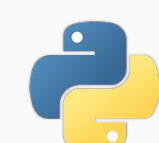

B

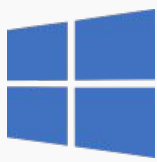

https://github.com/scikit-multiflow/scikit-multiflow
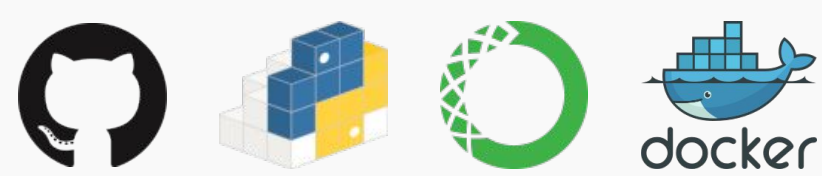


\section{How can I contribute?}

- We welcome contributions from the community

(P) scikit-multiflow

I||l gitter.im/scikit-multiflow/community

- We have a pool of projects in the following areas:

- Classification

- Regression

- Clustering

- Anomaly Detection

- Or bring your own project/idea

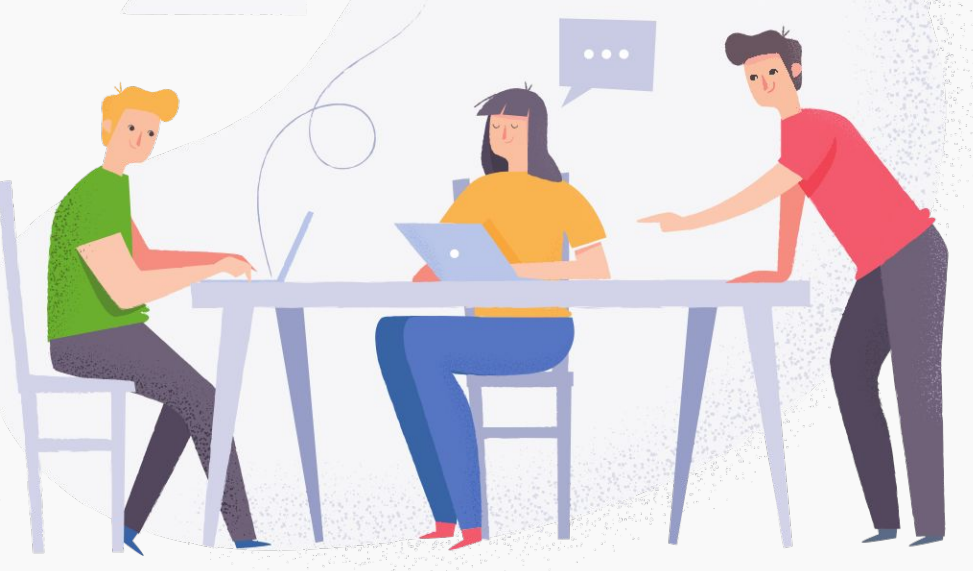




\section{Takeaways from this talk}

( Stream learning is an alternative to standard (batch) learning

- data is continuously generated

- data is non-stationary, it evolves! (concept drift)

( scikit-multiflow

- machine learning for data streams in Python

- easy to design and run experiments

- easy to extend 


\section{Thank you}

jacob.montiel [at] waikato.ac.nz<smiles>[CH]</smiles> 